\title{
Masses and Orbits of Extrasolar Planets: Preparation of Astrometric Observing Programs
}

\author{
Andreas Quirrenbach, Sabine Frink, \& David Mitchell \\ University of California San Diego, Center for Astrophysics and Space \\ Sciences, Mail Code 0424; La Jolla, CA, 92093-0424, USA
}

\begin{abstract}
Astrometry is the most powerful technique to determine dynamical masses and orbital parameters of extrasolar planets. The careful selection and characterization of reference stars is crucial for the success of any astrometric program. The best reference stars for NASA's Space Interferometry Mission (SIM) are distant $\mathrm{K}$ giants. The first sub-stellar companion to a $\mathrm{K}$ giant was recently detected serendipitously in a radialvelocity survey aimed at defining the SIM grid star strategy.
\end{abstract}

\section{Introduction}

The discovery of a planet orbiting the star 51 Pegasi (Mayor \& Queloz 1995) has opened a new field of astronomy: the study of extrasolar planetary systems. About 100 planets outside the Solar System are known to date, and new discoveries are announced almost every month. These developments have revolutionized our view of our own place in the Universe. We know now that other planetary systems can have a structure that is completely different from that of the Solar System. Moreover, the existential question whether other habitable worlds exist can for the first time in human history be addressed scientifically.

All known extrasolar planets have been found with an indirect technique, the radial-velocity (RV) method. What is actually detected is not the planet itself, but the motion of its parent star around the common center of gravity. The Doppler shift due to the line-of-sight component of this motion can be detected with spectroscopic methods. While RV surveys have had tremendous successes, it must not be forgotten that they have technical and astrophysical limitations, which necessarily lead to a biased view of exo-planetary astrophysics. It is therefore important to develop complementary techniques, which can give additional information on the systems already detected, and find planets in situations where the RV technique cannot be used.

\section{The Potential of Astrometry for Studies of Planetary Systems}

One of these complementary techniques is precise astrometry (e.g., Quirrenbach 2000). This approach is based on the same principle as the RV method, but it aims at measuring the two components of the parent star's reflex motion in the plane of the sky. Detection of planetary signatures requires an extraordinary precision (of order a few micro-arcseconds). An astrometric planet program can address the following outstanding issues: 
- Mass determination for planets detected in RV surveys (without the infamous $\sin i$ factor). The RV method gives only a lower limit to the mass, because the inclination of the orbit with respect to the line-of-sight remains unknown. Astrometry can resolve this ambiguity, because it measures two components of the orbital motion, from which the inclination can be derived.

- Confirmation of hints for long-period planets in RV surveys. Many of the stars with detected short-period planets also show long-term trends in the velocity residuals (Fischer et al. 2001). These are indicative of additional long-period planets, whose presence can be confirmed astrometrically.

- Inventory of planets around stars of all masses. The RV technique works well only for stars with a sufficient number of narrow spectral lines, i.e., fairly old stars with $M \lesssim 1.2 M_{\odot}$. Astrometry can detect planets around more massive stars and complete a census of gas and ice giants around stars of all masses.

- Detection of gas giants around pre-main-sequence stars, signatures of planet formation. Astrometry can detect giant planets around young stars, and thus probe the time of planet formation and migration. Observations of pre-mainsequence stars of different ages can provide a critical test of the formation mechanism of gas giants. Whereas gas accretion on $\sim 10 \mathrm{M}_{\oplus}$ cores requires $\sim 10 \mathrm{Myr}$, formation by disk instabilities would proceed rapidly and thus produce an astrometric signature even at very young stellar ages (Boss 1998).

- Detection of multiple systems with masses decreasing from the inside out. Whereas the astrometric signal increases linearly with the semi-major axis $a$ of the planetary orbit, the RV signal scales with $1 / \sqrt{a}$. This leads to opposite detection biases for the two methods. Systems in which the masses increase with $a$ (e.g., $v$ And, Butler et al. 1999) are easily detected by the RV technique because the planets' signatures are of similar amplitudes. Conversely, systems with masses decreasing with $a$ are more easily detected astrometrically.

- Determine whether multiple systems are coplanar or not. Many of the known extrasolar planets have highly eccentric orbits. A plausible origin of these eccentricities is strong gravitational interaction between two or several massive planets (Lin \& Ida 1997; Papaloizou \& Terquem 2001). This could also lead to orbits that are not aligned with the equatorial plane of the star, and to non-coplanar orbits in multiple systems.

- Search for massive terrestrial planets in the Solar neighborhood. NASA's Space Interferometry Mission (SIM) has a $1 \mu$ as precision goal in its "narrowangle" mode (within $1^{\circ}$ ). This opens the exciting perspective of looking for rocky planets down to a limit of a few Earth masses.

In summary, astrometry is a unique tool for dynamical studies of extrasolar planetary systems; its capabilities to determine masses and orbits are not matched by any other technique. Astrometric surveys of young and old planetary systems will give unparalleled insight into the mechanisms of planet formation, orbital migration and evolution, orbital resonances, and interaction between planets.

\section{Astrometric Reference Stars}

The success of SIM depends critically on the selection of suitable grid and reference stars. About 3,000 stars distributed evenly over the sky are needed for the grid. They must be stable (i.e., they must not show any motion except parallax 


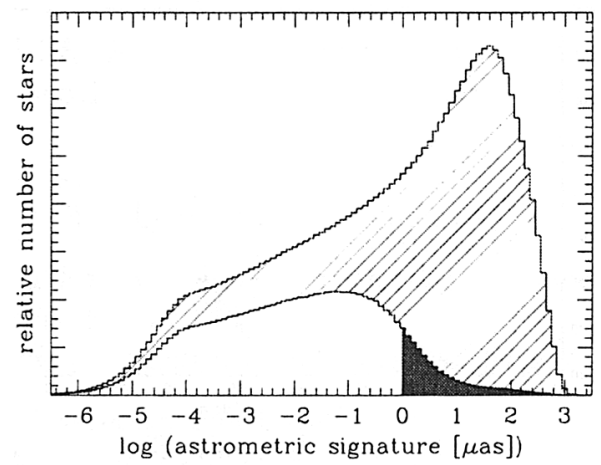

Figure 1. Distribution of the astrometric signatures as seen by SIM in a simulated binary input sample (hatched), among those binaries that would be missed in a RV survey (white), and among the subset of the latter with astrometric signatures larger than $1 \mu$ as (grey). This figure demonstrates the power of a RV survey in identifying the vast majority of stars that would cause major problems for the SIM grid.

and linear proper motion) on the level of a few $\mu$ as. For each narrow-angle target, at least three reference stars stable to $\sim 1 \mu$ as must be available within a $\sim 1^{\circ}$ search radius. Finding stars that meet these requirements is by no means a trivial task. They have to be identified and characterized before the launch of SIM, because the potential presence of even a fairly small number of unstable references can only be compensated by a dramatic increase in redundancy of the observations (Frink et al. 2001), which is very costly in terms of SIM time.

A key criterion for selecting suitable grid and reference stars for SIM is their distance $d$, because the wobble induced by planets or other unseen companions scales with $1 / d$. The best class of reference stars are therefore $\mathrm{K}$ giants, which are numerous even at high galactic latitudes, and intrinsically bright. Samples of candidate SIM reference stars can either be selected from existing astrometric catalogs, or identified in a specialized survey. The case for distant $\mathrm{K}$ giants as good grid stars rests on a three-fold argument:

- The wobble due to planetary companions is sufficiently small.

- We know from the RV surveys that brown dwarfs are rare as companions to $\mathrm{G}$ dwarfs, which are the progenitors of $\mathrm{K}$ giants. Brown-dwarf companions to $\mathrm{K}$ giants will therefore also be rare.

- Stellar companions can be detected efficiently before the launch of SIM by an RV survey. This is a non-trivial statement as photospheric activity could corrupt precise RV measurements. A survey with the Hamilton Echelle Spectrograph at Lick Observatory has shown, however, that many $\mathrm{K}$ giants are sufficiently stable; about $2 / 3$ of all $\mathrm{K}$ giants are drawn from a distribution with a mean of $\sim 20 \mathrm{~m} / \mathrm{s}$. A simulation shows that this allows the detection of most stellar companions with only two or three RV data points (Fig. 1).

It should thus be possible to define the SIM grid, and to gain confidence in its integrity, well before launch. The selection of narrow-angle reference stars is a more difficult problem because of the increased accuracy requirement and 


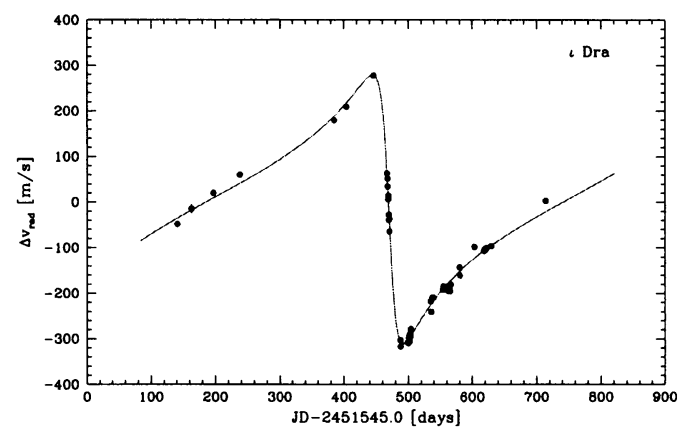

Figure 2. Radial-velocity variation of $\iota$ Dra over a period of two years, and best-fit Keplerian orbit.

more limited search area for suitable stars. Observations aimed at identifying reference stars for high-priority narrow-angle targets have recently been started.

\section{A Substellar Companion of the K Giant $\iota$ Dra}

In the course of our pilot RV survey of $\mathrm{K}$ giants, we serendipitously discovered a low-mass companion to $\iota$ Dra. A Keplerian fit to the data yields an orbital period of about 536 days and an eccentricity of 0.70 (Fig. 2). Assuming a mass of $1.05 M_{\odot}$ for $\iota$ Dra, the mass function implies a minimum companion mass of $8.9 M_{\text {jup }}$, making it a planet candidate. The corresponding semi-major axis is 1.3 AU. The non-detection of the orbital motion by HIPPARCos allows us to place an upper limit of $45 M_{\text {jup }}$ on the companion mass, establishing the substellar nature of the object. We estimate that transits in this system could occur already for inclinations as low as 81.5 , due to the large diameter of the giant star. The companion to $\iota$ Dra is the first brown dwarf or planet found to orbit a giant rather than a main-sequence star.

Acknowledgments. We thank P. Butler, D. Fischer, G. Marcy, S. Röser and E. Schilbach, without whom this work would not have been possible.

\section{References}

Boss, A. P. 1998, Nature, 393, 141

Butler, R. P., et al. 1999, ApJ, 526, 916

Fischer, D. A., et al. 2001, ApJ, 551, 1107

Frink, S., et al. 2001, PASP, 113, 173

Lin, D. N. C, \& Ida, S. 1997, ApJ, 477, 781

Mayor, M., \& Queloz, D. 1995, Nature, 378, 355

Papaloizou, J. C. B., \& Terquem, C. 2001, MNRAS, 325, 221

Quirrenbach, A. 2000, in ASP Conf. Ser. 213, Bioastronomy '99: A New Era in the Search for Life, ed. G. Lemarchand \& K. Meech, 119 